\title{
BiCMOS Technology Improvements for Microwave Application
}

\author{
Wibo D. van Noort ${ }^{l}$, A. Rodriguez ${ }^{1}$, HongJiang Sun ${ }^{1}$, Francis Zaato ${ }^{1}$, Nancy Zhang ${ }^{1}$, Tony Nesheiwat ${ }^{1}$, \\ Francois Neuilly', Joost Melai ${ }^{3}$, Erwin Hijzen ${ }^{4}$
}

\begin{abstract}
The third generation of NXP $0.25 \mu \mathrm{m}$ SiGe BiCMOS technology (QUBiC4Xi) is presented. The NPN has $f_{T} / f_{\max }$ of $216 / 177 \mathrm{GHz}$ and $B V_{\text {cb0 }}$ of $5.2 \mathrm{~V}$. The high-voltage NPN has 12 V BV $V_{c b 0}$, and $f_{T} / f_{\max }$ of 80/162 GHz. This is complemented with an improved MIM capacitor with $1 \mathrm{THz}$ cutoff frequency and new on-chip isolation structures that demonstrate a record $|\mathrm{S} 12|$ of $-60 \mathrm{~dB}$ at $10 \mathrm{GHz}$.

Index Terms-HBT, heterojunction bipolar transistor, substrate isolation, MIM capacitor, BiCMOS, bipolar, $\mathrm{Si}$, $\mathrm{SiGe}, \mathrm{SiGe}: \mathrm{C}, \mathrm{TaO}_{5}$.
\end{abstract}

\section{INTRODUCTION}

SiGe HBT technology and performance has been improved tremendously in the last decade. This has opened up new opportunities for silicon-based technology in the area of microwave and millimeter wave applications. However, these markets are not mature enough to support high wafer volumes and highly integrated solutions that are required for cost-effective manufacturing. Therefore, NXP has developed various $\mathrm{SiGe}$ process variants that are derived from the highly successful QUBiC4 $0.25 \mu \mathrm{m}$ BiCMOS technology family $[1,2]$. A high degree of commonality with the parent technology that is running in high volume is maintained in all cases. This ensures low cost of wafers and development, high manufacturability and control, and high quality that is proven in high volume.

Here an improved version of QUBiC4X is presented: (QUBiC4Xi) [2]. The technology comes with all the advanced passives of the parent technology and maintains full compatibility to the $0.25 \mu \mathrm{m}$ CMOS node $[1,3]$. Here we introduce a faster NPN heterojunction bipolar (HBT) that is discussed in Section II. A modified (single mask adder) $5 \mathrm{fF} / \mu \mathrm{m}^{2} \quad \mathrm{TaO}_{5}$ MIM capacitor with improved scalability and reduced top-plate resistance is presented in Section III. Section IV highlights the outstanding substrate isolation that can be achieved with the high-resistivity substrate $(200 \Omega \mathrm{cm}, \mathrm{CZ})$ and optimized layout techniques. We will finalize with some conclusions in the last section.

\section{THE NPN}

The bipolar device lies at the heart of the technology. The SiGe module has been implemented in such a way that most integration steps and mask levels can be shared with the (full-silicon) parent technology. The basic architecture is similar to the original concept [4]. Key aspects are the double poly architecture that ensures an excellent base link 1. NXP Semiconductors, PO Box 1279, Hopewell Junction, NY 12533, USA

wibo.van.noort@nxp.com.

2 NXP Semiconductors, Caen, France

3. Previously with NXP Research Leuven, now with MESA+,

University of Twente, the Netherlands.

4. NXP Semiconductors research, Leuven, Belgium and a non-self aligned approach that maintains process simplicity; it adds a mask but avoids a lot of critical process steps (e.g. selective epitaxy or critical CMP steps).

For this generation emphasis was placed on the base link region, the emitter-base spacer in particular and the $\mathrm{SiGe:C}$ layer stack.

The emitter-base region is shown schematically in Figure 1. The base link is determined by two factors: the emitter-base spacer and a patterned etch-stop layer to facilitate robust emitter-window patterning.

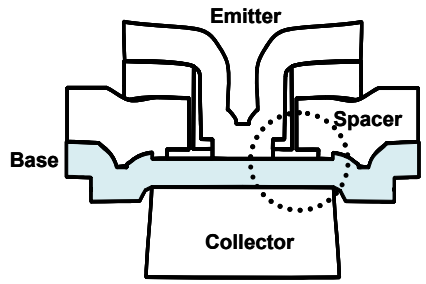

Figure 1 Schematic cross-section of the NPN device with the base-link area highlighted.

The spacer is most critical because it also determines the scalability of the emitter width. Figure 2 shows two TEM images that compare the new spacer module that was introduced to the original spacer.

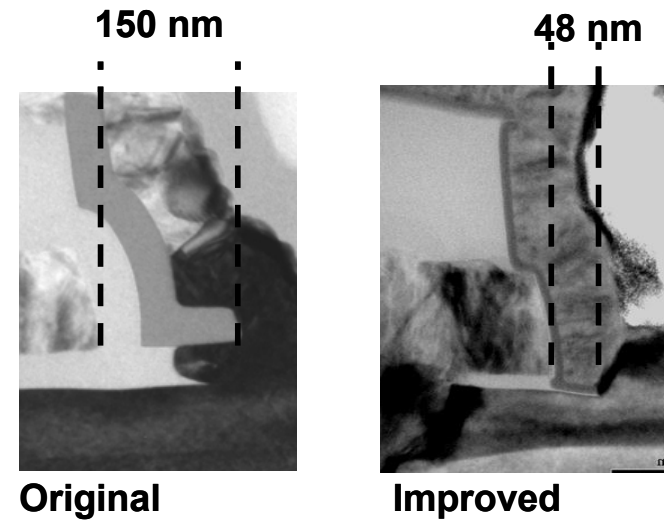

Figure 2 TEM image of original and improved emitterbase spacer.

The spacer width has been considerably reduced from 150 to $48 \mathrm{~nm}$. It is composed of a much thinner oxide nitride stack that is easier to integrate than the thicker layers.

The very thin layers form a nitride "blanket" that effectively seals off all the oxide layers. Wet etching solution cannot penetrate the tiny exposed oxide areas. This eliminates any undercut and allows for very aggressive precleaning prior to deposition of the epitaxial mono emitter. This leads to a high quality interface, very reproducible spacer dimensions and (effective) emitter width. 
The shorter distance under the spacer also leads to $50 \%$ reduction in base-link resistance as is shown in Figure 3. The improved scalability facilitates downscaling of the emitter width from $0.4 \mu \mathrm{m}$ to beyond $0.25 \mu \mathrm{m}$.

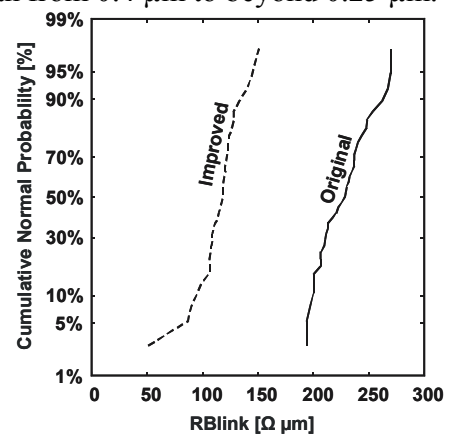

Figure 3 Cumulative probability of the base link resistance.

The SiGe base layer was also improved, a $30 \%$ reduction in base width and increased Ge content gives substantially higher current drive.

Table 1 shows parameters for a selection of scaled-down devices with the improved spacer module and the new SiGe stack. A "Fast" SiGe stack is also included in the first column. We consider the "slightly slower" stack to be a better tradeoff between base resistance and highvoltage/low voltage combination of devices.

\begin{tabular}{|c|c|c|c|c|c|c|}
\hline & \multicolumn{4}{|l|}{ NPN } & \multirow{3}{*}{ Comment } \\
\hline & & \multicolumn{3}{|l|}{ high freq } & \multirow[b]{2}{*}{ 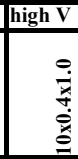 } & \\
\hline Area & $\mu \mathrm{m}^{2}$ & 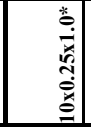 & 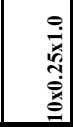 & 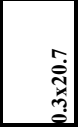 & & \\
\hline \multicolumn{2}{|l|}{ Annotation } & A1 & B1 & B2 & $\overline{C 1}$ & see figures \\
\hline$f_{T}$ & $\mathbf{G H z}$ & 216 & 176 & 163 & 80 & $\mathrm{~V}_{\mathrm{cb}}=1 \mathrm{~V}$ \\
\hline $\mathbf{f}_{\max }$ & GHz & 177 & 172 & 129 & 162 & $\mathrm{~V}_{\mathrm{cb}}=1 \mathrm{~V}$, unilateral gain \\
\hline slope & $\mathrm{dB} / \mathrm{dec}$ & $\begin{array}{ll}-19.7 \\
\end{array}$ & -19.3 & -19.9 & -20.7 & $\mathrm{~V}_{\mathrm{cb}}=1 \mathrm{~V}$, unilateral gain \\
\hline $\mathrm{C}_{\mathrm{be}}$ & $\mathrm{fF}$ & 45 & 37 & 66 & 47 & off state, $1 \mathrm{GHz}$ \\
\hline $\mathrm{C}_{\mathrm{cb}}$ & $\mathrm{fF}$ & 31 & 35 & 50 & 15 & off state, $1 \mathrm{GHz}$ \\
\hline $\mathrm{C}_{\mathrm{cs}}$ & $\mathrm{fF}$ & 8 & 8 & 6.25 & 12 & off state, $10 \mathrm{GHz}$ \\
\hline$\tau_{\mathrm{N}}$ & ps & 0.61 & 0.78 & 0.8 & 1.56 & on state, $13 \mathrm{GHz}$ \\
\hline$\left(\mathrm{C}_{\mathrm{be}}+\mathrm{C}_{\mathrm{cb}}\right)$ & fF & 105 & 95 & 224 & 76 & on state, $13 \mathrm{GHz}$ \\
\hline $\mathrm{BV}_{\mathrm{ce} 0}$ & $\mathrm{~V}$ & 1.44 & 1.44 & 1.44 & 2.5 & \\
\hline $\mathrm{BV}_{\mathrm{cb} 0}$ & $\mathrm{~V}$ & \begin{tabular}{l|l}
5.2 \\
\end{tabular} & 5.2 & 5.2 & 12 & \\
\hline Hfe & - & 2700 & 2700 & 2100 & 1700 & at $0.7 \mathrm{~V} \mathrm{Vbe}$ \\
\hline re & $\Omega$ & 3.4 & 14 & 2.9 & $<1$ & \\
\hline$I_{\mathrm{c} 0}$ & fA & 0.94 & 0.55 & 2 & 3.7 & \\
\hline Rpinch & $\mathrm{k} \Omega \mathrm{sq}$ & 4.8 & 3.25 & 3.25 & 3.25 & \\
\hline
\end{tabular}

Table 1 A selection of devices with some basic parameters. The first column is an alternative base layer with a different $\mathbf{r b} / \mathbf{f}_{\mathrm{T}}$ tradeoff.

The emitter has been divided into smaller islands to optimize the base resistance and to keep the power density (with regards to self-heating) in check. The total device size is large enough to allow for accurate RF characterization and deembedding.

All RF data presented was obtained from on-wafer measurements with an 8510 Agilent network analyzer (NWA) that is hooked up to a Cascade $12 \mathrm{k}$ semiautomatic probestation with Cascade $125 \mu \mathrm{m}$ pitch GSG Infinity probes. The system was calibrated with the customary
SOLT procedure. The measurement was subsequently deembedded with on-wafer "OPEN" and "SHORT" dummy structures.

Figure 4 shows the actual RF characteristics of the devices listed in Table 1. the highest $f_{T}(216 \mathrm{GHz})$ is obtained with the "fast" $4.8 \mathrm{k} \Omega / \mathrm{sq}$. stack. Nevertheless, the slower stack has identical $f_{\max }$ and a superior high-voltage device. This device is fabricated on the same wafer as the $176 \mathrm{GHz} f_{T}$ device. It has a remarkable combination of $12 \mathrm{~V}$ breakdown $\left(\mathrm{BV}_{\mathrm{cb} 0}\right)$ with an $f_{T} / f_{\max }$ of $80 / 162 \mathrm{GHz}$.
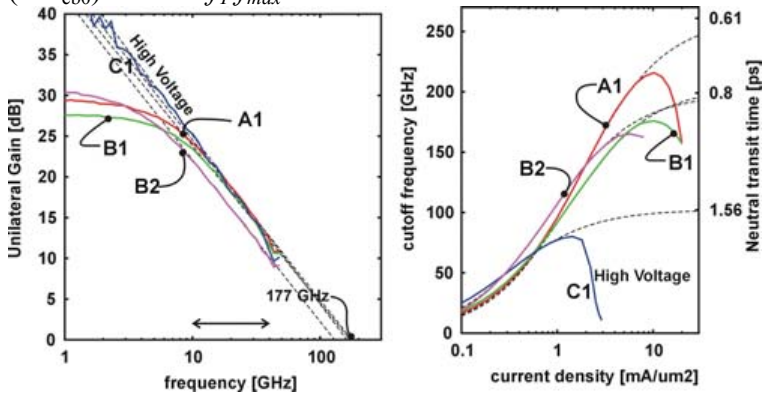

Figure 4 RF characteristics: unilateral gain vs frequency biased at maximum $f_{T}$ (left) and $f_{T}$ (|H21|*f@13 GHz) vs. Ic. All measurements were performed with $1 \mathrm{~V} \mathrm{Vcb}$. The annotation is relates to the detailed information in Table 1.

\section{METAL3 MIM CAPACITOR}

The $5 \mathrm{fF} / \mu \mathrm{m}^{2} \mathrm{MIM}$ capacitor in the parent technology is fabricated between metal 4 and metal 5 with a stack of TiN, $\mathrm{TaO}_{5}$ capped with another layer of TiN. It is implemented as a single-mask adder by virtue of optimized dry etching and cleaning procedures [5]. It is used extensively as an RF component and for decoupling.

High frequencies require much smaller capacitors, but the capacitance density must remain fixed as there will be a need for large decoupling capacitors regardless of the application frequency.

A smaller minimum geometry is the only solution. This can only be achieved in a lower metal level because the designrules for the very thick metal 4 and 5 and via 4 are very crude (i.e. Via4 size is $1 \mathrm{x} 1 \mu \mathrm{m}$, Metal 6 lines/spaces: $6 / 3 \mu \mathrm{m}$ ). Via 3 is more than 4 times smaller (in area), with a 3 times smaller pitch. A 4x smaller minimum size (that fits two vias) is achieved by moving the MIM layer from metal 4 to metal 3, reducing the minimum capacitance to $10 \mathrm{fF}$. A comparison of MIM position between parent technology and QUBiC4Xi is shown schematically in Figure 5.

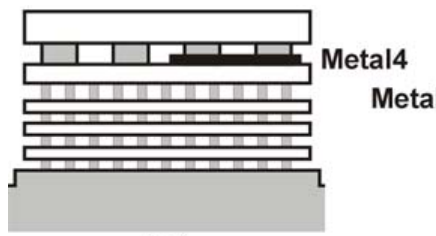

(a.)

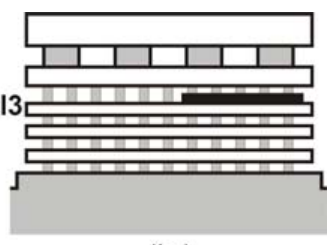

(b.)
Figure 5 Schematic figure showing MIM position in parent technology (a.) and QUBiC4Xi (b.). 
The RF quality factor $(\operatorname{Im}(\mathrm{Y} 12) / \operatorname{Re}(\mathrm{Y} 12)$ with $\mathrm{C}$ between port 1 and 2) is dominated by the series resistance imposed by limited conductivity of the TiN top plate. This was originally compensated with a thick TiN layer and a large number of vias connecting the top plate to the highly conductive metal 5 . Here we have added a layer of $\mathrm{AlCu}$ to the top plate and reduced the TiN thickness. The overall stack is: metal 3, TiN, $\mathrm{TaO}_{5}, \mathrm{TiN}$, capped by $\mathrm{AlCu}$. The higher conductivity of $\mathrm{AlCu}$ vs. TiN allows for a thinner overall stack with a $10 \mathrm{x}$ reduction in top-plate sheet resistance.

The RF performance up to $50 \mathrm{GHz}$ of various geometries is shown in Figure 6.

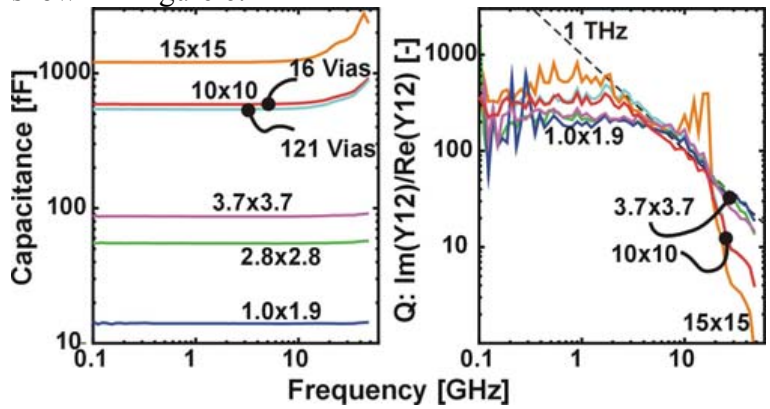

Figure 6 Measured RF performance of 6 MIM designs. Capacitance (left plot) is $\operatorname{Im}(-Y 12) / 2 \pi f$, $Q$ factor (right) the ratio between imaginary and real part of $Y 12$.

All capacitors have an excellent bandwidth, over a large range of capacitance values. The larger values are impacted above $10 \mathrm{GHz}$ or so by miniscule parasitic inductance $(\sim 5 \mathrm{pH})$.

The quality factor (as defined for a capacitor) follows a limit corresponding to a $1 \mathrm{THz}$ cutoff. The large capacitors are in close proximity to self resonance at high frequency. This increases the real part of Y12 that is unrelated to RF losses (resistance) and thus defeats the $\mathrm{Q}$ definition that assumes pure capacitive behavior.

The parasitic capacitance to the substrate is obviously increased (from 5.55 to $7.77 \mathrm{aF} / \mu^{2}$ ) by moving down one level. Nevertheless, it is still $\sim 3$ orders of magnitude smaller than the capacitance itself $\left(5 \mathrm{fF} / \mu \mathrm{m}^{2}\right)$; negligible in both cases.

\section{SUBSTRATE ISOLATION}

Isolation is a major challenge in electronic circuits and becomes especially challenging in analog/RF IC design due to close proximities, high dynamic range, high frequency and a shared silicon substrate.

There are various design aspects that play a crucial role in achieving high isolation. Three concepts discussed here are illustrated in Figure 7.

The signal pads are connected to the underlying P-Well with Buried P or N-Well with Buried N (as shown in the cross section). The substrate is a $(200 \mathrm{~mm}) 200 \Omega \mathrm{cm}$ P-type $\mathrm{CZ}$ wafer; standard material for $\mathrm{QUBiC} 4+$ and all $\mathrm{SiGe}$ derivatives $[1,2]$.

In the first structure, isolation is provided by several rings of deep-trench isolation (DTI) around the well (a. in the figure). The second structure is similar but has an additional conductive, grounded guard ring around each pad (b. in the figure). The third variant is similar to the second, but now the ground domains between the two ports have been disconnected (c. in the picture).

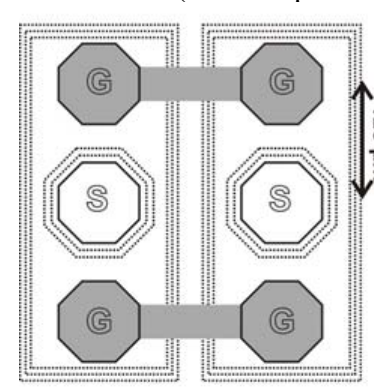

(a.)

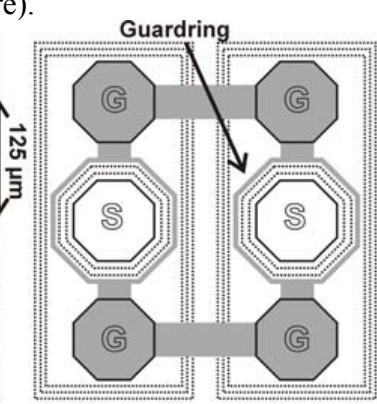

(b.)

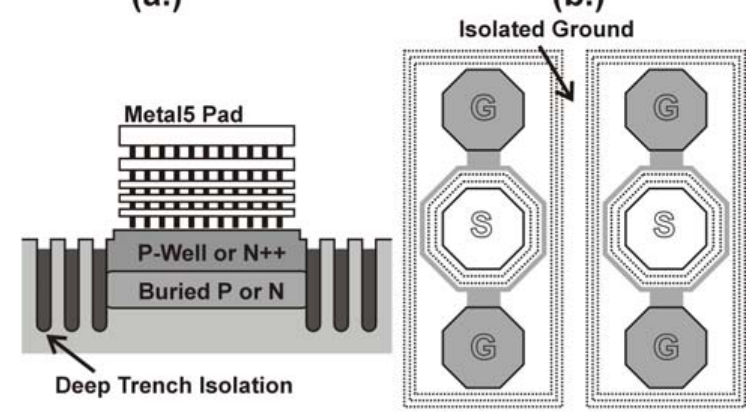

(c.)

Figure 7 Schematic drawing of the layout of isolation GSG test structures. The signal pad is connected to a P-type or N-type area (lower left crossection). Three concepts: DTI isolation (a.), DTI isolation with a grounded guardring (b.), DTI isolation with a grounded gurdring with isolated ground domains.

The layouts are designed in such a way that they fit directly to on-wafer (125 $\mu \mathrm{m}$ pitch) GSG probes. This way open/short deembedding is avoided, which would be problematic with very poor isolation of "OPEN", and sometimes, "SHORT" dummy. Consequently, results shown in this section are non-deembedded values taken straight from the (calibrated) NWA.

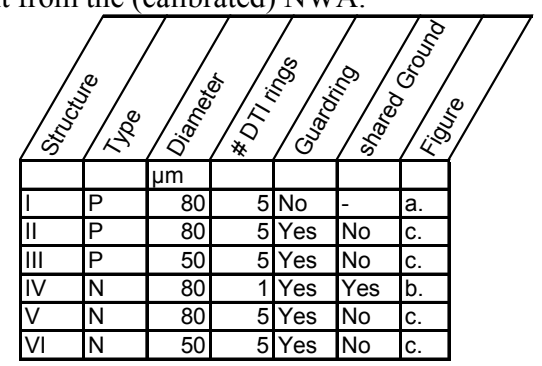

Table 2 A selection of isolation structures with reference to Figure 7.

RF measurements of the selection of structures presented in Table 2 are shown in Figure 8 and Figure 9.

The high degree of isolation that can be achieved with properly designed guardrings in separate ground domains is 
remarkable. To our knowledge these values exceed anything previously reported [6,7]. Furthermore, it is surprising, counterintuitive, to see equivalent isolation between P-type and N-type connections above $1 \mathrm{GHz}$ or so. This is clearly the high substrate resistivity at work.

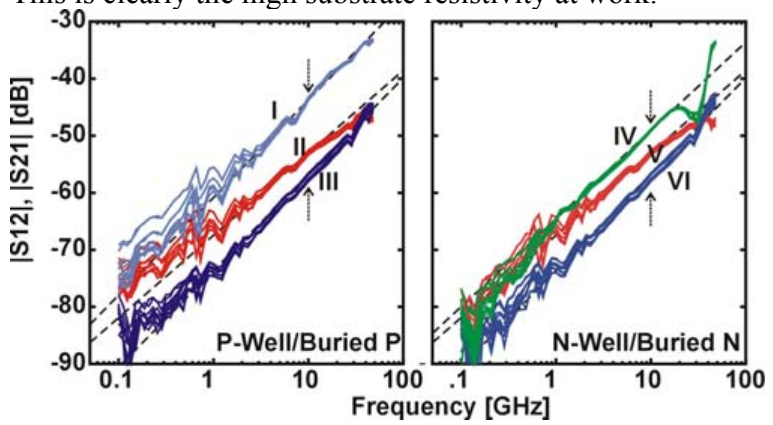

Figure 8 Isolation for structures in Table 2 in terms of |S12|, |S21| with $50 \Omega$ system impedance. Left plot: Ptype, right plot: $\mathrm{N}$-type.

The behavior is predominantly capacitive with a slope slightly deviating from $20 \mathrm{~dB} / \mathrm{dec}$, which can be explained by distributed $\mathrm{RC}$ effects. It is somewhat arbitrary to present the results in terms of S parameter magnitude. The isolation between actual circuit nodes will depend on their impedance level, which is likely to be different from the $50 \Omega$ system impedance.

To complement the picture, the input impedance of the isolated pads also needs to be considered. Note that in the particular case of isolation the transfer coefficients are much smaller than the reflection coefficients thus resulting in $\mathrm{Y} 11 \approx 1 / \mathrm{Z} 11$ and $\mathrm{Y} 22 \approx 1 / \mathrm{Z} 22$ (with $\mathrm{Y}$ and $\mathrm{Z}$ following the usual two-port definitions). Hence the apparent arbitrary choice of inverse input/output impedance in Figure 9 reflects the general case very well.

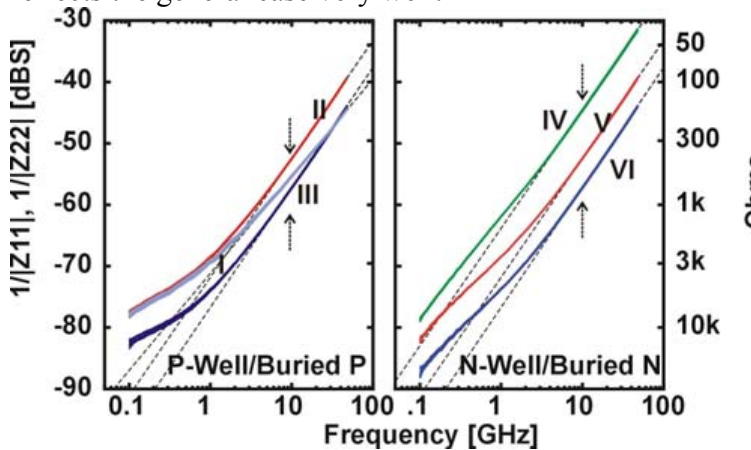

Figure 9 Inverse input impedance of structures in Table 2 in $\mathbf{d B}($ Siemens). Left plot: P-type, right plot: $\mathrm{N}$-type.

The impedance exhibits capacitive behavior at high frequencies. Below $1 \mathrm{GHz}$ the direct $\mathrm{DC}$ connection $(\sim 30 \mathrm{k} \Omega$ resistance $)$ between the P-type pads becomes noticeable.

The pads without guardring (I), despite poor isolation, have a very decent input impedance al the way up to $50 \mathrm{GHz}$. The guardring with a single DTI ring (IV) reaches unacceptable low impedance already at $10 \mathrm{GHz}$. The structures with 5 DTI rings and a guard ring (II, III, V, VI) have a considerably higher input impedance combined with high isolation.

The pad diameter also plays an important role, the smaller pads (50 $\mu \mathrm{m}$ diameter) have superior isolation that scales roughly with the perimeter at high frequency $(20 \cdot \log (80 / 50) \sim 4 \mathrm{~dB})$.

\section{CONCLUSIONS}

A highly versatile and low-cost $0.25 \mu \mathrm{m} \mathrm{SiGe:C} \mathrm{microwave}$ technology is presented. It features a scaled NPN with cutoff frequency of $216 \mathrm{GHz}$ and $177 \mathrm{GHz} \mathrm{f}_{\max }$. A high-voltage device with $12 \mathrm{~V} \mathrm{BV}_{\mathrm{cb} 0}, 80 \mathrm{Ghz} \mathrm{f}_{\mathrm{T}}$ and $162 \mathrm{GHz} \mathrm{f}_{\max }$ is also supported. The $5 \mathrm{fF} / \mu \mathrm{m}^{2} \mathrm{MIM}$ capacitor is very similar to that of the parent technology but with improved scalability and versatility by introducing smaller designrules and higher top-plate conductivity. It has ample RF performance with a cutoff frequency (frequency where $\mathrm{Q}$ reaches 1) of $\sim 1 \mathrm{THz}$ and a bandwidth in excess of $10 \mathrm{GHz}$ for a $1 \mathrm{pF}$ capacitor.

On-chip isolation of $-60 \mathrm{~dB}$ (S12 magnitude) of a $50 \mu \mathrm{m}$ diameter N-Well or P-Well island is demonstrated, this is among the highest values reported. The highest isolation is achieved with a combination of deep trench isolation and guardrings with isolated ground domains.

\section{ACKNOWLEDGEMENTS}

We are very grateful for support by Engineering and Failure analysis groups at NXP Semiconductors East Fishkill. We also acknowledge support form IMEC P-Line.

\section{REFERENCES}

[1] Deixler, P. Letavic, T. Mahatdejkul, T. Bouttement, Y. Brock, R. Tan, P.C. Saikumar, V. Rodriguez, A. Colclaser, R. Kellowan, P. Sun, H. Bell, N. Bower, D. Yao, A. van Langevelde, R. Vanhoucke, T. van Noort, W.D. Hurkx, G.A.M. Crespo, D. Biard, C. Bardy, S. Slotboom, J.W. "QUBiC4plus: a cost-effective BiCMOS manufacturing technology with elite passive enhancements optimized for 'silicon-based' RF-system-inpackage environment" in: Proceedings of the 2005 Bipolar/BiCMOS Circuits and Technology Meeting, pp. 272-5, 2005.

[2]P. Deixler et.al., "QUBiC4X: An fT/fmax $=130 / 140 \mathrm{GHz}$ SiGe:CBiCMOS manufacturing technology with elite passives for emerging microwave applications", in: Proceedings of the 2004 Bipolar/BiCMOS Circuits and Technology Meeting, pp. 233-6, Sept 2004.

[3] van Noort, Wibo D. Detchevery, C. Rodriguez, A. Pijper, R., "On-chip mm-Wave passives", in: Proceedings of the 2007 Bipolar/BiCMOS Circuits and Technology Meeting, pp. 268-71, 2007.

[4] Deixler, P. Colclaser, R. Bower, D. Bell, N. De Boer, W. Szmyd, D. Bardy, S. Wilbanks, W. Barre, P. v Houdt, M. Paasschens, J.C.J. Veenstra, H. v d Heijden, E. Donkers, J.J.T.M. Slotboom, J.W "QUBiC4G: a f/sub $\mathrm{T} / / \mathrm{f} / \mathrm{sub} \max /=70 / 100 \mathrm{GHz} 0.25 / \mathrm{spl} \mathrm{mu} / \mathrm{m}$ low power SiGe-BiCMOS production technology with high quality passives for $12.5 \mathrm{~Gb} / \mathrm{s}$ optical networking and emerging wireless applications up to $20 \mathrm{GHz}$, in: Proceedings of the 2002 Bipolar/BiCMOS Circuits and Technology Meeting, pp. 201-4, 2002.

[5] Hongjiang Sun, Eyup Aksen, Ka Man Lau, Nancy Bell, "Fully Integrated Tantalum Pentoxide Metal-insulator-Metal capacitors for $\mathrm{Si}$ and SiGe RF-BiCMOS Technologies" in: Conference Proceedings AMC XIX, Materials Research Soc., 2003.

[6] Martin Pfost, Hans-Martin Rein, "Modeling and Measurement of Substrate Coupling in Si-Bipolar IC's up to $40 \mathrm{GHz}$ ", IEEE Journal of Solid State Circuits, pp. 582-91, vol. 33, No. 4, April 1998.

[7] D. Szmyd, L. Gambus, A. Wilbanks, "Strategies and test structures for improving isolation between circuit blocks" in: Proceedings of the 2002 International Conference on Microelectronic Test Structures, 2002. ICMTS 2002. pp. 89-93, April 2002 